\title{
Fractional Linear Multistep Methods for Abel-Volterra Integral Equations of the Second Kind
}

\author{
By Ch. Lubich
}

\begin{abstract}
Fractional powers of linear multistep methods are suggested for the numerical solution of weakly singular Volterra integral equations. The proposed methods are convergent of the order of the underlying multistep method, also in the generic case of solutions which are not smooth at the origin. The stability properties (stability region, $A$-stability, $A(\alpha)$-stability) are closely related to those of the underlying linear multistep method.
\end{abstract}

1. Introduction. We consider the Abel integral equation of the second kind,

$$
\begin{aligned}
y(t)=f(t)+\frac{1}{\Gamma(\alpha)} \int_{0}^{t}(t-s)^{\alpha-1} g(s, y(s)) d s \text { in } \mathbf{R}^{n} \\
\quad t \in I:=[0, T], \text { with fixed } \alpha>0 .
\end{aligned}
$$

The case $\alpha=\frac{1}{2}$ is encountered in a variety of problems in physics and chemistry; see, e.g., the references in [2], [19]. So far, numerical methods for (1) have usually been based on the concept of product integration ([1], [2], [3], [4] and the references given there). In the present work we suggest an entirely different approach.

We consider convolution quadratures

$$
y_{n}=f\left(t_{n}\right)+h^{\alpha} \sum_{j=0}^{n} \omega_{n-j}^{(\alpha)} g\left(t_{j}, y_{j}\right)+h^{\alpha} \sum_{j=0}^{m} w_{n j} g\left(t_{j}, y_{j}\right), \quad n h \in I,
$$

where $h$ is the stepsize, and $t_{n}=n h$. The convolution quadrature weights $\omega_{n}^{(\alpha)}$ and the starting quadrature weights $w_{n j}(j=0, \ldots, m ; m$ fixed $)$ are independent of $h$. For the computation of $y_{0}, \ldots, y_{N}$ one thus needs $O(N) f$ - and $g$-evaluations and only $O\left(N(\log N)^{2}\right)$ arithmetic operations if Fast Fourier Transform techniques as in [7] are used.

In the special case $\alpha=1$ and $f(t) \equiv y_{0}$, Eq. (1) reduces to the ordinary differential equation $y^{\prime}=g(t, y), y(0)=y_{0}$. If a linear multistep method $(\rho, \sigma)$ (where, as usual, $\rho$ and $\sigma$ denote the generating polynomials of the method, see [8]) is applied to this initial-value problem, the method can be rewritten as a convolution quadrature (2) with weights $\omega_{n}$ given via their generating power series $\omega(\zeta)=\sum_{0}^{\infty} \omega_{n} \zeta^{n}$ by

$$
\omega(\zeta)=\sigma(1 / \zeta) / \rho(1 / \zeta)
$$

see [17], [21], [13].

Received January 12, 1984; revised November 27, 1984.

1980 Mathematics Subject Classification. Primary 65R20; Secondary 65L05. 
The present work deals with the following question: Given a linear multistep method $\omega=(\rho, \sigma)$, can we use it to construct a convolution quadrature (2) for arbitrary $\alpha$, which has the same convergence properties and the same (or even better) stability properties as the original multistep method? Under appropriate conditions on $(\rho, \sigma)$ the answer is positive and surprisingly simple:

For the linear multistep method $\omega=(\rho, \sigma)$ we assume

The method is stable and consistent of order $p$. The method is implicit, and all zeros of $\sigma(\zeta)$ have absolute value $\leqslant 1$.

We define a convolution quadrature $\omega^{\alpha}=\left(\omega_{n}^{(\alpha)}\right)_{0}^{\infty}$ via its generating power series $\omega^{\alpha}(\zeta)=\sum_{0}^{\infty} \omega_{n}^{(\alpha)} \zeta^{n}$ by

$$
\omega^{\alpha}(\zeta)=\omega(\zeta)^{\alpha}
$$

This can be interpreted as the linear multistep method $\omega=(\rho, \sigma)$ taken to the fractional power $\alpha$. Such convolution quadratures, called fractional linear multistep methods, have been introduced by the author in [15]. A different approach is contained in [16]. In Sections 2 and 3 we show that $\omega^{\alpha}$ has the desired convergence and stability properties. Fractional linear multistep methods are simple to implement. In Section 4 we give a numerical example for a fractional backward differentiation method.

2. Convergence. Instead of Eq. (1) we consider in this section the slightly more general integral equation

$$
y(t)=f(t)+\frac{1}{\Gamma(\alpha)} \int_{0}^{t}(t-s)^{\alpha-1} K(t, s, y(s)) d s \quad \text { in } \mathbf{R}^{n}, t \in I=[0, T]
$$

for fixed $\alpha>0$, under the following smoothness assumptions:

$K(t, s, y)$ is sufficiently differentiable on $\{(t, s, y) ; 0 \leqslant s \leqslant t \leqslant$

$\left.T, y \in \mathbf{R}^{n}\right\}, f(t)=F\left(t, t^{\alpha}\right)$ where $F\left(t_{1}, t_{2}\right)$ is sufficiently differentiable.

The solution $y(t)$ is then unique on its nonempty existence interval, which is assumed to contain all of $I$. It can be written in the form (see [12])

$$
\begin{aligned}
& y(t)=Y\left(t, t^{\alpha}\right) \text { for some sufficiently differentiable function } \\
& Y\left(t_{1}, t_{2}\right) .
\end{aligned}
$$

Usually $y(t)$ is not smooth at $t=0$.

We study the discretization (with notations as in (2))

(9) $y_{n}=f\left(t_{n}\right)+h^{\alpha} \sum_{j=0}^{n} \omega_{n-j}^{(\alpha)} K\left(t_{n}, t_{j}, y_{j}\right)+h^{\alpha} \sum_{j=0}^{m} w_{n j} K\left(t_{n}, t_{j}, y_{j}\right), \quad n h \in I$.

We have the following convergence result.

THEOREM 1. (Convergence of fractional linear multistep methods.) Let the linear multistep method $\omega=(\rho, \sigma)$ satisfy (4), and consider $\omega^{\alpha}$ defined by (5). One can construct a starting quadrature $w_{n j}=O\left(n^{\alpha-1}\right)$ ( see (10) below) such that the error of the numerical solution $y_{n}$, given by (9), for the exact solution $y(t)$ of $(6)$, (7) satisfies

$$
\left|y_{n}-y(t)\right| \leqslant C \cdot t^{\beta-1} \cdot h^{p} \quad(t=n h),
$$

where the constant $C$ does not depend on $n$ and $h$ with $n h \leqslant T, h \leqslant \bar{h}(\bar{h}>0$ sufficiently small). The exponent $\beta$ satisfies $\beta>\alpha$, and, in particular, $\beta \geqslant 1$ for $\alpha=\frac{1}{2}, \frac{2}{3}, \frac{3}{4}, \frac{4}{5}, \ldots$. 
Remark. If $y(t)$ is actually smooth at $t=0$, we can take $\beta=1$ for all $\alpha>0$.

Proof. The proof is based on the results in [15].

(a) Consistency error. We begin with the construction of the starting quadrature. Let $A=\{\gamma=k+l \alpha ; k, l \geqslant 0$ integer, $\gamma \leqslant p-1\}$ and card $A=m+1$. We choose $w_{n j}(n \geqslant 0 ; j=0,1, \ldots, m)$ such that for all exponents $\gamma \in A$,

$$
\begin{aligned}
& h^{\alpha} \sum_{j=0}^{n} \omega_{n-j}^{(\alpha)}(j h)^{\gamma}+h^{\alpha} \sum_{j=0}^{m} w_{n j}(j h)^{\gamma} \\
& \quad=\frac{1}{\Gamma(\alpha)} \int_{0}^{t}(t-s)^{\alpha-1} s^{\gamma} d s \quad(t=n h) .
\end{aligned}
$$

Then $w_{n j}$ are well-defined, independent of $h$ and of magnitude $O\left(n^{\alpha-1}\right)$, see [15]. With the techniques of [15, Theorem 2.4] it follows that, for every function $\varphi(t)=\Phi\left(t, t^{\alpha}\right)$, with $\Phi\left(t_{1}, t_{2}\right)$ sufficiently smooth, we have

$$
\begin{aligned}
& h^{\alpha} \sum_{j=0}^{n} \omega_{n-j}^{(\alpha)} \varphi(j h)+h^{\alpha} \sum_{j=0}^{m} w_{n j} \varphi(j h) \\
& \quad=\frac{1}{\Gamma(\alpha)} \int_{0}^{t}(t-s)^{\alpha-1} \varphi(s) d s+O\left(t^{\beta-1} \cdot h^{p}\right)
\end{aligned}
$$

uniformly for $n h=t \leqslant T$, where

(12) $\beta=\alpha-p+1+\min \{\gamma=k+l \alpha ; k, l \geqslant 0$ integer, $\gamma>p-1\}>\alpha$.

The constant in the $O\left(t^{\beta-1} \cdot h^{p}\right)$ term of (11) can be estimated by bounds on a finite number of derivatives of $\Phi$. So the consistency error, given at $t=n h$ by

$$
\begin{aligned}
d_{n}=\mid h^{\alpha} \sum_{j=0}^{n} \omega_{n-j}^{(\alpha)} K\left(t_{n}, t_{j}, y\left(t_{j}\right)\right) & +h^{\alpha} \sum_{j=0}^{m} w_{n j} K\left(t_{n}, t_{j}, y\left(t_{j}\right)\right) \\
& -\frac{1}{\Gamma(\alpha)} \int_{0}^{t}(t-s)^{\alpha-1} K(t, s, y(s)) d s \mid,
\end{aligned}
$$

can be estimated, because of (8) and (11), by

$$
d_{n} \leqslant M_{0} t^{\beta-1} \cdot h^{p}=M_{0} \cdot n^{\beta-1} \cdot h^{p+\beta-1} \quad(n h=t \leqslant T)
$$

for some constant $M_{0}$.

(b) Error propagation. Let $L$ denote a (local) Lipschitz constant of $K$. For the global error of (9),

we then have

$$
e_{n}=\left|y_{n}-y\left(t_{n}\right)\right|
$$

$$
e_{n} \leqslant d_{n}+h^{\alpha} L\left(\sum_{j=0}^{n}\left|\omega_{n-j}^{(\alpha)}\right| e_{j}+\sum_{j=0}^{m}\left|w_{n j}\right| e_{j}\right) .
$$

From [15] we have

$$
\omega_{n}^{(\alpha)}=O\left(n^{\alpha-1}\right), \quad \text { and also } w_{n j}=O\left(n^{\alpha-1}\right) \text { for } j=0,1, \ldots, m .
$$

Further, it is known (cf. [6, p. 47]) that

$$
\frac{n^{\mu-1}}{\Gamma(\mu)}=\left(1+O\left(n^{-1}\right)\right) \cdot(-1)^{n}\left(\begin{array}{c}
-\mu \\
n
\end{array}\right) \text { for fixed } \mu>0
$$

Also,

$$
(-1)^{n}\left(\begin{array}{c}
-\mu \\
n
\end{array}\right)>0 \text { for all } n \geqslant 0 \text {, if } \mu>0
$$


Taking $\mu=\beta$ in (13) and $\mu=\alpha$ in (14) we obtain

$$
e_{n} \leqslant M \cdot h^{p+\beta-1} \cdot(-1)^{n}\left(\begin{array}{c}
-\beta \\
n
\end{array}\right)+h^{\alpha} c L \sum_{j=0}^{n}(-1)^{n-j}\left(\begin{array}{c}
-\alpha \\
n-j
\end{array}\right) e_{j}
$$

for some constants $M$ and $c$. Hence,

$$
e_{n} \leqslant M \cdot h^{p} \cdot u_{n},
$$

where $u_{n}$ is the $n$th coefficient of the power series $u_{h}(\zeta)=\sum_{0}^{\infty} u_{n} \zeta^{n}$ defined by

$$
u_{h}(\zeta)=h^{\beta-1}(1-\zeta)^{-\beta}+c L^{\alpha}(1-\zeta)^{-\alpha} u_{h}(\zeta)
$$

i.e.,

$$
u_{h}(\zeta)=\frac{1}{h} U\left(\frac{1-\zeta}{h}\right) \text { with } U(z)=\frac{z^{-\beta}}{1-c L z^{-\alpha}}
$$

$U(z)$ is the Laplace transform of the function $u(t)$, given as the solution of the linear integral equation

$$
u(t)=\frac{t^{\beta-1}}{\Gamma(\beta)}+c L \int_{0}^{t} \frac{(t-s)^{\alpha-1}}{\Gamma(\alpha)} u(s) d s, \quad t>0 .
$$

It is known from [20, Theorem XXI] (the Post-Widder inversion formula) that

$$
u_{n} \rightarrow u(t) \text { as } h \rightarrow 0 \text { for fixed } t=n h .
$$

One has actually the estimate, proved (by more elementary means) in [16, Theorem 10.1],

$$
u_{n}-u(t)=O\left(t^{\beta-2} \cdot h\right) \quad \text { uniformly for } n h=t \leqslant T .
$$

This implies, in particular,

$$
u_{n} \leqslant C \cdot t^{\beta-1} \quad(t=n h),
$$

where the constant $C$ is independent of $h$ (sufficiently small) and $n$ with $n h \leqslant T$. Inserting (16) in (15) completes the proof.

Remark. Concerning estimates for the error propagation in terms of the Lipschitz constant of the kernel (part (b) of the above proof), fractional linear multistep methods do not differ from previously existing numerical methods. Theorem 1 could also be proved by using (in (b)) techniques as in [9, Theorem 5.1]. The reference to the presently unpublished paper [16] could thus be omitted. An $O\left(h^{q}\right)$ error estimate, with $q=\min (p, p+\beta-1)$, would also be obtained with the elementary proof of Theorem 2 in [11].

We conclude this section with a remark on the error constant $c^{*}$. For a linear multistep method $\omega=(\rho, \sigma), c^{*}$ is given by

$$
h \omega\left(e^{-h}\right)=1+c^{*} h^{p}+O\left(h^{p+1}\right) .
$$

For the fractional linear multistep method $\omega^{\alpha}$ we have, therefore,

$$
h^{\alpha} \omega^{\alpha}\left(e^{-h}\right)=1+\alpha c^{*} h^{p}+O\left(h^{p+1}\right) .
$$

For $\omega^{\alpha}$, the constant $\alpha c^{*}$ plays a similar role as the error constant $c^{*}$ for $\omega$ (this is implicit in [15, Section 3]). We continue to call $c^{*}$ the error constant of $\omega^{\alpha}$. 
3. Stability. In many applications, Abel equations (1) are stable as $t \rightarrow \infty$; see, e.g., [10], [19]. One would then wish stable behavior also for the discretized equation (2), and, in particular, for the propagation of the error. As in [14], we study the linearized equation

$$
y(t)=f(t)+\frac{\lambda}{\Gamma(\alpha)} \int_{0}^{t}(t-s)^{\alpha-1} y(s) d s, \quad t \geqslant 0,0<\alpha<1 .
$$

For $|\arg \lambda-\pi|<(1-\alpha / 2) \pi$ the solution of (17) satisfies

$y(t) \rightarrow 0$ as $t \rightarrow \infty$ whenever $f(t)$ converges to a finite limit, and $y(t)$ is bounded whenever $f(t)$ is bounded.

Applying a convolution quadrature (2) to (17) yields

$$
y_{n}=f_{n}+h^{\alpha} \lambda \sum_{j=0}^{n} \omega_{n-j}^{(\alpha)} y_{j}, \quad n \geqslant 0 .
$$

Here $f_{n}=f\left(t_{n}\right)+h^{\alpha} \lambda \sum_{j=0}^{m} w_{n j} y_{j}$. We assume that $w_{n j}=O\left(n^{\alpha-1}\right)$, so that $\left(f_{n}\right)$ converges (is bounded) if $f(t)$ converges as $t \rightarrow \infty$ (is bounded, resp.).

As in [14], we extend the classical stability concepts for linear multistep methods to convolution quadratures $\omega^{\alpha}$ :

The stability region $S$ is the set of all $z=h^{\alpha} \lambda$ for which the numerical solution $\left(y_{n}\right)$ given by $(18)$ satisfies

$$
y_{n} \rightarrow 0 \text { as } n \rightarrow \infty \text { whenever }\left(f_{n}\right) \text { converges to a finite limit. }
$$

The method is $A$-stable if $S$ contains the analytical stability region $|\arg z-\pi|<$ $(1-\alpha / 2) \pi$.

The method is $A(\varphi)$-stable if $S$ contains the sector $|\arg z-\pi|<\varphi$.

It is well-known that the stability region of a linear multistep method $\omega=(\rho, \sigma)$ equals $\mathbf{C} \backslash\{\rho(\zeta) / \sigma(\zeta) ;|\zeta| \geqslant 1\}=\mathbf{C} \backslash\{1 / \omega(\zeta) ;|\zeta| \leqslant 1\}$. An identical characterization holds for $\omega^{\alpha}$.

THEOREM 2. The stability region of a fractional linear multistep method (4), (5) is given by

$$
S=\mathbf{C} \backslash\left\{1 / \omega^{\alpha}(\zeta) ;|\zeta| \leqslant 1\right\} .
$$

Proof. The proof is based on Theorem 3 of [14]. Let $\zeta_{i}(i=0, \ldots, r)$ and $\tilde{\zeta}_{j}$ $(j=0, \ldots, s)$ denote the zeros of absolute value 1 of $\rho(\zeta)$ and $\sigma(\zeta)$, respectively. By (4) we then have

$$
\omega^{\alpha}(\zeta)=\prod_{i=0}^{r}\left(1-\zeta_{i} \zeta\right)^{-\alpha} \cdot \prod_{j=0}^{s}\left(1-\tilde{\zeta}_{j} \zeta\right)^{\alpha} \cdot u(\zeta),
$$

where $u(\zeta)$ is holomorphic in a neighborhood of $|\zeta| \leqslant 1$. The coefficients of $u(\zeta)$ and $\left(1-\tilde{\zeta}_{j} \zeta\right)^{\alpha}$ are in $l^{1}$, hence also the coefficients of the product of these power series. Therefore,

$$
\omega^{\alpha}(\zeta)=\prod_{i=0}^{r}\left(1-\zeta_{i} \zeta\right)^{-\alpha} v(\zeta),
$$

where the coefficients of $v(\zeta)$ are in $l^{1}$. If $\zeta_{0}=1$ is the only zero of absolute value 1 of $\rho(\zeta)$, we can apply Theorem 3 of [14]. Otherwise, a straightforward generalization of that theorem gives the result. 
Remark. If $h^{\alpha} \lambda \in S$, then the solution of (18) also satisfies:

$\left(y_{n}\right)$ is bounded whenever $\left(f_{n}\right)$ is bounded [14, Corollary 5].

As an immediate corollary we obtain the following.

THEOREM 3. Let the linear multistep method $\omega=(\rho, \sigma)$ satisfy (4), and consider $\omega^{\alpha}$ defined by (5). Let $S_{\omega}$ and $S_{\omega^{\alpha}}$ denote the stability regions of $\omega$ and $\omega^{\alpha}$, respectively. Then, we have

(a) $\left(\mathbf{C} \backslash S_{\omega^{\alpha}}\right)=\left(\mathbf{C} \backslash S_{\omega}\right)^{\alpha}$.

(b) $\omega^{\alpha}$ is $A$-stable if and only if $\omega$ is $A$-stable.

(c) With $\pi-\varphi=\alpha(\pi-\psi), \omega^{\alpha}$ is $A(\varphi)$-stable if and only if $\omega$ is $A(\psi)$-stable.

Example. The $k$-step backward differentiation formula (BDFk) satisfies for $k \leqslant 6$ assumption (4) with $p=k$ and is $A(\psi)$-stable for some $\psi>0$, see [18]. For $\alpha=\frac{1}{2}$, the fractional BDF method (BDFk $)^{1 / 2}$ is therefore $A(\pi / 2)$-stable for all $k \leqslant 6$.

Combining part (b) with Theorem 2.2 in Dahlquist [5] yields

THEOREM 4. The order, $p$, of an A-stable fractional linear multistep method cannot exceed 2. The smallest error constant, $c^{*}=1 / 12$, is obtained for the fractional trapezoidal rule, defined by

$$
\omega^{\alpha}(\zeta)=\left(\frac{1}{2} \frac{1+\zeta}{1-\zeta}\right)^{\alpha}
$$

4. Numerical Example. The integral equation

$$
y(t)=-\frac{1}{\sqrt{\pi}} \int_{0}^{t}(t-s)^{-1 / 2}(y(s)-\sin s)^{3} d s
$$

arises from a parabolic boundary value problem in the theory of superfluidity [10]. We have applied the (BDF4) $)^{1 / 2}$ method (fourth-order backward differentiation formula to power $1 / 2$, see [15, Example 2.8]) to this equation. The quadrature weights were computed as described in [15]. The numerical results at $t=8$ are given in Table 1. The exact solution is $y(8)=0.3236412904$. This value has been obtained numerically using various methods with small step sizes. The computations were done in FORTRAN double precision on the IBM 370/168 of the University of Heidelberg.

TABLE 1

\begin{tabular}{l|c|c}
\hline \multicolumn{1}{c|}{$h$} & numerical solution & error \\
\hline 0.1 & 0.3236520328 & $1.07_{10}-5$ \\
0.05 & 0.3236421096 & $8.19_{10}-7$ \\
0.025 & 0.3236413206 & $3.02_{10}-8$ \\
\hline
\end{tabular}

Acknowledgment. The author wishes to thank E. Hairer for helpful discussion.

Institut für Mathematik und Geometrie

Universität Innsbruck

Technikerstrasse 13

A-6020 Innsbruck, Austria 
1. C. T. H. BAKer, The Numerical Treatment of Integral Equations, Clarendon Press, Oxford, 1977.

2. H. BRUNNER, "A survey of recent advances in the numerical treatment of Volterra integral and integro-differential equations," J. Comput. Appl. Math., v. 8, 1982, pp. 213-229.

3. H. BRUNNER, "The numerical solution of integral equations with weakly singular kernels," in Numerical Analysis (D. F. Griffiths, ed.), Proceedings Dundee 1983, Lecture Notes in Math., vol. 1066. Springer-Verlag, Berlin and New York, 1984.

4. R. F. Cameron \& S. McKeE, "Product integration methods for second-kind Abel integral equations," J. Comput. Appl. Math., v. 11, 1984, pp. 1-10.

5. G. Dahlquist, “A special stability problem for linear multistep methods," BIT, v. 3, 1963, pp. $27-43$.

6. A. ErdEl YI (editor), Higher Transcendental Functions, vol. I, McGraw-Hill, New York, 1953.

7. E. Hairer, Ch. Lubich \& M. Schlichte, "Fast numerical solution of nonlinear Volterra convolution equations," SIAM J. Sci. Statist. Comput. (To appear.)

8. P. Henrici, Discrete Variable Methods in Ordinary Differential Equations, Wiley, New York, 1962.

9. D. KERSHAw, "Some results for Abel-Volterra integral equations of the second kind," in Treatment of Integral Equations by Numerical Methods (C. T. H. Baker and G. F. Miller, eds.), Academic Press, London, 1982, pp. 273-282.

10. N. LeVInSON, "A nonlinear Volterra equation arising in the theory of superfluidity," J. Math. Anal. Appl., v. 1, 1960, pp. 1-11.

11. P. LiNZ, "Numerical methods for Volterra integral equations with singular kernels," SIAM J. Numer. Anal., v. 6, 1969, pp. 365-375.

12. Сн. Lubich, "Runge-Kutta theory for Volterra and Abel integral equations of the second kind," Math. Comp., v. 41, 1983, pp. 87-102.

13. Сн. Lubich, "On the stability of linear multistep methods for Volterra convolution equations," IMA J. Numer. Anal., v. 3, 1983, pp. 439-465.

14. Сн. LUBich, "A stability analysis of convolution quadratures for Abel-Volterra integral equations," IMA J. Numer. Anal. (To appear.)

15. CH. Lubich, “Discretized fractional calculus,” SIAM J. Math. Anal. (To appear.)

16. Сн. Lubich, Discretized Operational Calculus. Part I: Theory, Technical Report, Inst. f. Math. u. Geom., Univ. Innsbruck, 1984. (Submitted for publication.)

17. J. Matrhys, “ $A$-stable linear multistep methods for Volterra integro-differential equations,” Numer. Math., v. 27, 1976, pp. 85-94.

18. S. P. NørSetr, "A criterion for $A(\alpha)$-stability of linear multistep methods," $B I T$, v. 9, 1969, pp. 259-263.

19. W. E. Olmstead \& R. A. Handelsman, "Diffusion in a semi-infinite region with nonlinear surface dissipation," SIAM Rev., v. 18, 1976, pp. 275-291.

20. E. L. Post, “Generalized differentiation,” Trans. Amer. Math. Soc., v. 32, 1930, pp. 723-781.

21. P. H. M. Wolkenfelt, The Numerical Analysis of Reducible Quadrature Methods for Volterra Integral and Integro-Differential Equations, Thesis, Math. Centrum, Amsterdam, 1981. 